\title{
MicroRNA-124 rs531564 Polymorphism and Cancer Risk: A Meta-analysis
}

\author{
Wen-Jing Li ${ }^{1,2}$, Yong Wang ${ }^{1}$, Yu Gong $^{1}$, Chao Tu${ }^{1}$, Tong-Bao Feng ${ }^{1}$, Chun-Jian Qi ${ }^{1 *}$
}

\begin{abstract}
Several studies reported there was a polymorphism $(r 5531564 \mathrm{C}>\mathrm{G})$ in miR-124 gene. To investigate the MiR-124 rs531564 polymorphism and cancer risk. We conducted a literature search of the Medline, Embase and Wangfang Medicine databases to identify all relevant studies for this meta-analysis. We determined that the miR-124 rs531564 polymorphism was significantly associated with decreased risks of cancers in the allelic model (G vs $\mathrm{C}, \mathrm{OR}=0.71,95 \% \mathrm{CI}=0.53-0.94, P=0.02)$, homozygote model (GG vs $\mathrm{CC}, \mathrm{OR}=0.42,95 \% \mathrm{CI}=\mathbf{0 . 2 6 - 0 . 6 6}$, $P=0.0002$ ), dominant model (GG/GC vs $\mathrm{CC}, \mathrm{OR}=0.71,95 \% \mathrm{CI}=\mathbf{0 . 5 1 - 0 . 9 8 ,} \boldsymbol{P = 0 . 0 4 )}$ ) and recessive model (GG vs $\mathrm{GC} / \mathrm{CC}, \mathrm{OR}=0.43,95 \% \mathrm{CI}=\mathbf{0 . 2 7 - 0 . 6 9}, P=0.0004)$. In an analysis stratified by cervical cancer group, significant

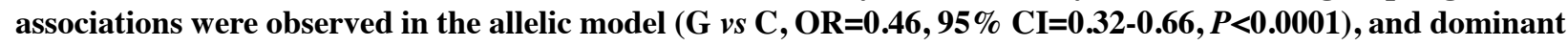

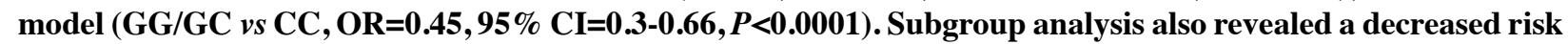
for esophageal squamous cell carcinoma in the homozygote model (GG vs CC, OR=0.45, 95\% CI $=0.27-0.75$, $P=0.002)$ and recessive model $(\mathrm{GG} v \mathrm{GC} / \mathrm{CC}, \mathrm{OR}=0.46,95 \% \mathrm{CI}=0.28-0.75, P=0.002)$. This meta-analysis suggests that the miR-124 rs531564 $\mathrm{C}>\mathrm{G}$ polymorphism is an important risk factor for cancers among the Chinese population.
\end{abstract}

Keywords: miR-124 - rs531564 - polymorphism - cancer risk - meta-analysis

Asian Pac J Cancer Prev, 16 (17), 7905-7909

\section{Introduction}

MicroRNAs (miRNAs) are an abundant class of small, single-stranded, nonprotein-coding RNAs measuring approximately 18-25 nucleotides in length that are highly evolutionarily conserved and negatively regulate gene expression by complementary base pairing with thousands of target mRNAs in the 3'-untranslated regions (3'UTR), which leads to mRNA cleavage or translational repression (Ambros, 2004; He and Hannon, 2004; Bartel, 2009; Ebert and Sharp, 2012; Fabian and Sonenberg, 2012). A single miRNA can bind to as many as 200 gene targets, and one-third of human genes, including cancer-associated genes, are regulated by miRNAs (He and Hannon, 2004). Many studies have indicated that there are strong links between miRNAs and human cancers. Researchers have demonstrated that some miRNAs located in chromosomal regions that are amplified in cancers can function as oncogenes, while miRNAs located in regions that are deleted in cancers might act as tumor suppressors (Kong et al., 2012). It has been shown that miRNAs are related to various critical biological processes, such as cell proliferation, apoptosis, differentiation, migration, metabolism, angiogenesis, signal transduction and tumor development and progression (Suarez and Sessa, 2009; Hatley et al., 2010; Krol et al., 2010; Kutanzi et al., 2011; Jansson and Lund, 2012; Kong et al., 2012; Pritchard et al., 2012; Ji et al., 2014). Most miRNAs are located at cancer-associated genomic regions or fragile sites, and aberrant miRNA gene expression plays a critical role in carcinogenesis (Calin and Croce, 2006; Esquela-Kerscher and Slack, 2006). Although the exact mechanism of miRNA deregulation in cancers remains unknown, some key dysregulated miRNAs have already been used as molecular biomarkers (Ruan et al., 2009). Li et al. reported miR-124 might be a tumor suppressor in breast cancer that functions via the regulation of FLOT1 ( $\mathrm{Li}$ et al., 2013). Moreover, Furuta et al. found that miR-124 and miR-203 are novel tumor-suppressive miRNAs for hepatocellular carcinoma that re epigenetically silenced and activate multiple targets during hepatocarcinogenesis (Furuta et al., 2010).

Single-nucleotide polymorphisms (SNPs) in microRNA genes might alter microRNA expression and maturation and might also further alter the effects of microRNAs on their target genes (Link et al., 2012; Pasquinelli, 2012). Common single-nucleotide polymorphisms (SNPs) in pre-miRNAs and cancer risk has been investigated in casecontrol studies in the last decade, and some common SNPs in pre-miRNAs have been demonstrated to be associated with increased cancer risk, including the hsa-miR-196a2 rs11614913 (Gao et al., 2011; Wang et al., 2012) and hsamiR-146a rs2910164 (Lian et al., 2012; Wang et al., 2012) polymorphisms. Because some miRNAs appear to have

${ }^{1}$ Medical Research Center, the Affiliated Hospital of Nanjing Medical University, Changzhou No.2 People's Hospital, ${ }^{2}$ Department of Oncology, Wujin People’s Hospital, Changzhou, China*For correspondence: qichunjian@njmu.edu.cn 
their own promoters, SNPs in the promoter region might play critical roles in the development of human cancers.

A single-nucleotide polymorphism (SNP, rs531564) has been identified in the miR-124 gene. In recent years, some case-control studies have been conducted to determine the associations between rs531564 and multiple types of cancers, such as esophageal cancer (Yin et al., 2013; Zhang et al., 2014a) and cervical cancer (Wu and Zhang, 2014; Xiong et al., 2014), but the results are conflicting rather than conclusive. Therefore, we conducted a meta-analysis of all relevant published studies to obtain a more accurate evaluation of the association between the miR124 rs531564 polymorphism and cancer risk.

\section{Materials and Methods}

\section{Publication search}

We performed searches of the Medline, Embase and Wangfang Medicine databases for all possible case-control studies, and we used the following keywords and subject terms: "miR-124 or miRNA-124 or microRNA-124 or rs531564"; "polymorphism or polymorphisms or variant or variants or SNP"; and "cancer or carcinoma". The literature search was last updated on the 27th of February 2015. There was no language limitation in the literature search. All eligible studies were retrieved, and their bibliographies were checked for other relevant publications. Review articles and the bibliographies of other relevant studies identified were searched by hand to identify additional eligible studies. The eligible studies had to meet all of the following criteria: (a) full-text study, (b) evaluation of the association between miRNA polymorphisms and cancer risk, (c) unrelated case-control design, and (d) sufficient data for estimating the odds ratio (OR) with $95 \%$ confidence intervals (CIs) and a $P$-value. Studies containing two or more case-control groups were considered as two or more independent studies.

\section{Data extraction}

Two investigators independently reviewed and extracted the information from all publications that met the inclusion criteria. In cases of conflict, an agreement was reached by discussion between the two reviewers. The following information was sought from each publication: the first author's surname, year of publication, country of origin, ethnicity, cancer type, genotyping method, source of control groups, numbers of cases and controls for each genotype.

\section{Statistical analyses}

The departure of the frequency of the miR-124 polymorphism from the expectations of Hardy-Weinberg equilibrium (HWE) was assessed via a chi-square test in the controls, and $P<0.05$ was considered to indicate significant disequilibrium. The crude OR corresponding to the $95 \%$ CI was used to assess the strength of the associations between the miRNA polymorphisms and cancer risks according to the methods published (Woolf, 1955). The statistical significance of the pooled OR was determined with a Z-test, and a $P$-value of $<0.05$ was considered statistically significant. For miR-124 G/C, we investigated the association between the genetic variants and cancer risks with an allelic comparison $(\mathrm{G}$ vs $\mathrm{C}$ ), a heterozygote model ( $\mathrm{GC}$ vs $\mathrm{CC}$ ), a homozygote model (GG vs CC), a dominant model (GG/GC vs CC), and recessive model (GG vs GC/CC). Subgroup analyses were also conducted by cancer type. When heterogeneity was absent $(p>0.10)$, the pooled OR was estimated by the fixed-effects model (the Mantel-Haenszel method) (Mantel and Haenszel, 1959); otherwise, the randomeffects model (the DerSimonian and Laird method) was employed (DerSimonian and Laird, 1986). Publication bias was detected with a Begg's funnel plot and Egger's linear regression method, and $p<0.05$ was considered statistically significant (Egger et al., 1997).

All statistical analyses were performed with Review Manager Software 5.2 (Cochrane Collaboration, Oxford, UK).

\section{Results}

\section{Study characteristics}

A total of 75 articles were achieved in the Medline, Embase and Wangfang Medicine databases. As shown in Figure 1,26 studies were retrieved for detailed evaluation. After screening the abstract, 49 studies were excluded, and 26 were selected and evaluated in detail. Seventeen of those 26 studies were excluded based on the inclusion criteria. Ultimately, 4 articles with 2, 003 cases and 2, 429 controls were included in the meta-analysis (Yin et al., 2013; Wu and Zhang, 2014; Xiong et al., 2014; Zhang et al., 2014a). The main characteristics of the studies are presented in Table 1. All of the studies performed in Chinese populations. Among the studies, there were two that were related to esophageal squamous cell carcinoma (Yin et al., 2013; Zhang et al., 2014a) and another two that were related to cervical cancer (Wu and Zhang, 2014; Xiong et al., 2014). The distributions of genotypes in the controls in all studies were in agreement with HWE.

\section{Main results}

The association between the miR-124 rs531564

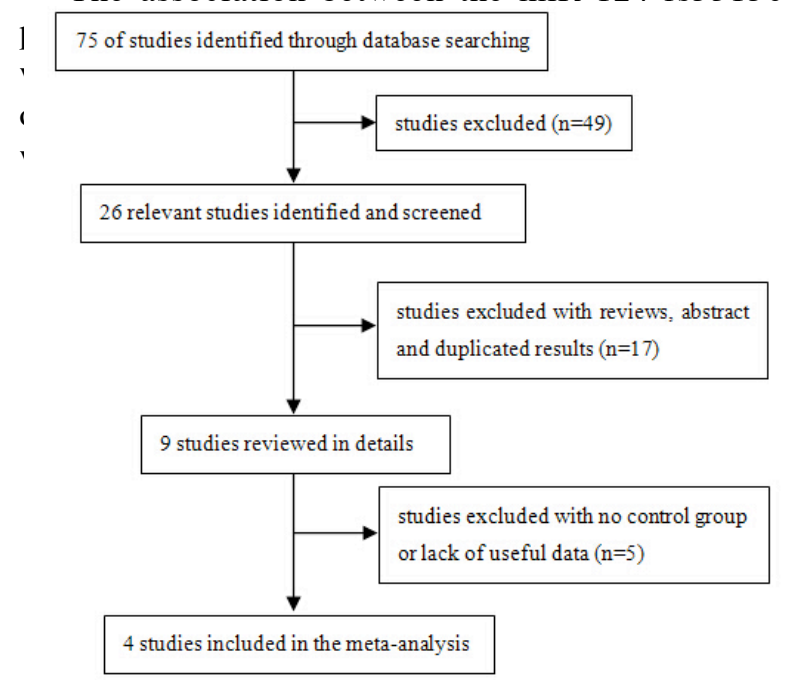

Figure 1. Flow Chart of the Studies and Specific Reasons for Exclusion from the Meta-Analysis 
Table 1. Characteristics of the Studies in the Meta-analysis

\begin{tabular}{|c|c|c|c|c|c|c|c|c|c|c|c|}
\hline$\overline{\text { Autor }}$ & Year & Country & Ethnicity & y Cancer type G & Genotyping & Design & No. of $\mathrm{Ge}$ & 1otypes di & ribution $\mathrm{o}$ & $\overline{\text { ses/contro }}$ & $\overline{\operatorname{HWE}(\mathrm{P})}$ \\
\hline & & & & & methods & & cases/controls & $\mathrm{CC}$ & $\overline{C G}$ & $\overline{\mathrm{GG}}$ & \\
\hline$\overline{\text { Zhang }}$ & 2014 & China & Asian & ESCC & PCR & PB & $629 / 686$ & $803 / 910$ & $295 / 331$ & $11 / 34$ & $\mathrm{p}=0.55$ \\
\hline Yin & 2013 & China & Asian & ESCC & PCR & $\mathrm{HB}$ & $1109 / 1275$ & $454 / 470$ & $146 / 168$ & $11 / 19$ & $\mathrm{p}=0.4$ \\
\hline Xiong & 2014 & China & Asian & Cervical Cancer & r PCR & $\mathrm{PB}$ & $107 / 208$ & $91 / 151$ & $15 / 51$ & $1 / 6$ & $\mathrm{p}=0.51$ \\
\hline $\mathrm{Wu}$ & 2014 & China & Asian & Cervical Cancer & r PCR & PB & $158 / 260$ & $134 / 184$ & $22 / 66$ & $2 / 10$ & $\mathrm{p}=0.19$ \\
\hline
\end{tabular}

HB: hotpial based; PB: population based; ESCC: esophageal squamous cell carcinoma; HWE: Hardy-Weinberg equilibrium; P: $p$ value

Table 2. Stratification Analyses of the Genetic Susceptibility to Cancer

\begin{tabular}{|c|c|c|c|c|c|c|c|c|}
\hline & & \multicolumn{3}{|c|}{ Test of association } & \multicolumn{3}{|c|}{ Test of heterogeneity } & \multirow[b]{2}{*}{ Model } \\
\hline & & OR $[95 \% \mathrm{CI}]$ & $\mathrm{Z}$ & p-value & $\chi^{\wedge} 2$ & $\mathrm{p}$-value & $\mathrm{I}^{\wedge} 2(\%)$ & \\
\hline \multirow[t]{5}{*}{ Total } & $\mathrm{G} v s \mathrm{C}$ & $0.71[0.53,0.94]$ & 2.38 & 0.02 & 11.16 & 0.01 & 73 & $\mathrm{R}$ \\
\hline & $\mathrm{GC} v s \mathrm{CC}$ & $0.75[0.54,1.04]$ & 1.74 & 0.08 & 11.34 & 0.01 & 74 & $\mathrm{R}$ \\
\hline & GG vs CC & $0.42[0.26,0.66]$ & 3.69 & 0.0002 & 1.45 & 0.69 & 0 & $\mathrm{~F}$ \\
\hline & $\mathrm{GG} / \mathrm{GC}$ vs $\mathrm{CC}$ & $0.71[0.51,0.98]$ & 2.07 & 0.04 & 11.91 & 0.008 & 75 & $\mathrm{R}$ \\
\hline & $\mathrm{GG} v s \mathrm{GC} / \mathrm{CC}$ & $0.43[0.27,0.69]$ & 3.55 & 0.0004 & 1.31 & 0.73 & 0 & $\mathrm{~F}$ \\
\hline \multirow[t]{5}{*}{ ESCC } & $\mathrm{G} v s \mathrm{C}$ & $0.88[0.78,1.01]$ & 1.86 & 0.06 & 0.12 & 0.73 & 0 & $\mathrm{~F}$ \\
\hline & $\mathrm{GC} v s \mathrm{CC}$ & $0.97[0.84,1.13]$ & 0.38 & 0.7 & 0.52 & 0.47 & 0 & $\mathrm{~F}$ \\
\hline & GG vs CC & $0.45[0.27,0.75]$ & 3.09 & 0.002 & 0.9 & 0.34 & 0 & $\mathrm{~F}$ \\
\hline & $\mathrm{GG} / \mathrm{GC}$ vs $\mathrm{CC}$ & $0.92[0.8,1.07]$ & 1.1 & 0.27 & 0.32 & 0.57 & 0 & $\mathrm{~F}$ \\
\hline & $\mathrm{GG} v s \mathrm{GC} / \mathrm{CC}$ & $0.46[0.28,0.75]$ & 3.06 & 0.002 & 1.01 & 0.31 & 1 & $\mathrm{~F}$ \\
\hline \multirow[t]{5}{*}{ Cervical Cancer } & $\mathrm{G} v s \mathrm{C}$ & $0.46[0.32,0.66]$ & 4.2 & $<0.0001$ & 0.03 & 0.86 & 0 & $\mathrm{~F}$ \\
\hline & $\mathrm{GC} v s \mathrm{CC}$ & $0.47[0.32,0.69]$ & 3.83 & 0.0001 & 0.02 & 0.89 & 0 & $\mathrm{~F}$ \\
\hline & $\mathrm{GG} v s \mathrm{CC}$ & $0.45[0.26,0.76]$ & 2.99 & 0.003 & 0.46 & 0.5 & 0 & $\mathrm{~F}$ \\
\hline & $\mathrm{GG} / \mathrm{GC}$ vs $\mathrm{CC}$ & $0.45[0.3,0.66]$ & 4.03 & $<0.0001$ & 0.03 & 0.86 & 0 & $\mathrm{~F}$ \\
\hline & $\mathrm{GG} v s \mathrm{GC} / \mathrm{CC}$ & $0.32[0.09,1.11]$ & 1.8 & 0.07 & 0 & 0.99 & 0 & $\mathrm{~F}$ \\
\hline
\end{tabular}

ESCC: esophageal squamous cell carcinoma; F: fixed-effects model; R: random-effects model

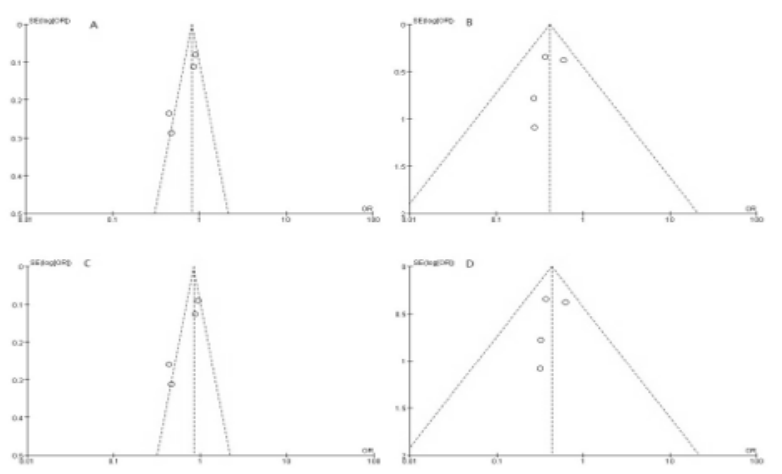

Figure 2. Funnel Plot for the Publication Bias Test. Each circle denotes an independent study of the indicated association. $\log [\mathrm{OR}]$, natural logarithm of the OR. The horizontal line indicates the mean effect size. A) $\mathrm{G}$ versus $\mathrm{C}$; B) $\mathrm{GG}$ versus $\mathrm{CC}$; C) $\mathrm{GG} / \mathrm{GC}$ versus CC; D) GG versus GC/CC

$p>0.10$, the pooled OR was estimated by the fixedeffects model; otherwise, the random-effects model was employed. The results of the pooled analysis revealed that decreased risks were observed in the comparisons of the allelic model $(\mathrm{G}$ vs $\mathrm{C}, \mathrm{OR}=0.71,95 \% \mathrm{CI}=0.53-0.94$, $P=0.02$ ), homozygote model (GG vs $\mathrm{CC}, \mathrm{OR}=0.42,95 \%$ $\mathrm{CI}=0.26-0.66, P=0.0002)$, dominant model $(\mathrm{GG} / \mathrm{GC} v s$ $\mathrm{CC}, \mathrm{OR}=0.71,95 \% \mathrm{CI}=0.51-0.98, P=0.04)$ and recessive model (GG vs $\mathrm{GC} / \mathrm{CC}, \mathrm{OR}=0.43,95 \% \mathrm{CI}=0.27-0.69$, $P=0.0004)$. Subgroup analyses by cancer type indicated that the miR-124 polymorphism might decrease the risk of ESCC in the homozygote model (GG vs CC, OR=0.45, 95\% $\mathrm{CI}=0.27-0.75, P=0.002)$ and recessive model (GG vs $\mathrm{GC} / \mathrm{CC}, \mathrm{OR}=0.46,95 \% \mathrm{CI}=0.28-0.75, P=0.002)$. In the cervical cancer group, significant associations were observed in the allelic model $(\mathrm{G} v s \mathrm{C}, \mathrm{OR}=0.46,95 \%$ $\mathrm{CI}=0.32-0.66, P<0.0001)$, heterozygote model (GC vs CC, $\mathrm{OR}=0.47,95 \% \mathrm{CI}=0.32-0.69, P=0.0001)$, homozygote model (GG $v s \mathrm{CC}, \mathrm{OR}=0.45,95 \% \mathrm{CI}=0.26-0.76, P=0.003$ ) and dominant model (GG/GC vs $\mathrm{CC}, \mathrm{OR}=0.45,95 \%$ $\mathrm{CI}=0.3-0.66, P<0.0001)$.

\section{Publication bias}

We used funnel plots and to access the publication bias of the literature. The results indicated that no significant publication biases were detected in the Begg's funnel plot of any of the comparison models. Symmetrical funnel plots were obtained for the SNP that was tested in homozygote model and the recessive model (Figure 2).

\section{Discussion}

miRNAs are a class of small, single-stranded non-coding RNAs that are involved in important biological processes related to apoptosis, proliferation, differentiation, angiogenesis, and the immune response (Ruan et al., 2009). SNPs are the most common genetic sequence variations in the human genome and can influence cancer susceptibility in the population. The dysfunction of miRNAs might have profound effects on the expression of numerous genes that might possibly contribute to cancer susceptibility (Ahn et al., 2013; Dikeakos et al., 2014; Tong et al., 2014). Single-nucleotide polymorphisms (SNPs) in microRNAs gene might alter microRNA expression and maturation and might also further change the effects of microRNAs on their target genes. SNPs in miRNAs are regarded as key factors in 
oncogenesis. Previous studies have demonstrated that miR-124 functions as a tumor suppressor in some cancers. For example, miR-124 expression is significantly reduced in gastric cancer, colon, prostate, breast cancer, cervical cancer, and bladder cancer. miR-124 can arrest the cell cycle and restrain the growth of bladder cancer by directly targeting CDK4 (Zhang et al., 2014b). In gastric cancer, a low expression level of miR-124 is also correlated with proliferation, migration, and invasion of tumor cells, and miR-124 can act as a tumor suppressor by targeting ROCK1 (Hu et al., 2014). Recent studies have shown that miR-124 exerts its tumor suppressing activity via the regulation of target genes such as amotL1 (Wan et al., 2014), Foxq1 (Peng et al., 2014), Ets-1 (Li et al., 2014) and SMC4 (Jinushi et al., 2014). These findings mean that the up-regulation of miR-124 might contribute to decreased cancer risk via the down-regulation of these targets.

Some researchers have examined the association of the miR-124 rs531564 polymorphism with the risks of several cancers observed significant relationships (Yin et al., 2013; Wu and Zhang, 2014; Xiong et al., 2014; Zhang et al., 2014a). The aim of our study was to demonstrate the relationship of the miR-124 rs531564 polymorphism with cancer risks using meta-analysis. We found that miR-124 rs531564 was associated with significantly decreased cancer risks in the in the allelic model $(\mathrm{G} v s \mathrm{C})$, homozygote model (GG vs CC), dominant model (GG/ $\mathrm{GC}$ vs CC) and recessive model (GG vs GC/CC). When stratified by cancer type, we observed a similar result for esophageal squamous cell carcinoma and cervical cancer. These findings suggest, for the first time, that the GG homozygote rs531564 polymorphism might have a potentially protective role against cancer in the Chinese population. Of course, we must noted that our study population was small; therefore, it is still necessary to examine larger sample studies to consider gene-gene and gene-environment interactions and using standardized unbiased genotyping methods, homogeneous gastric cancer patients, and sufficiently matched controls.

However, considering the limited studies and small population sizes included in our meta-analysis, our results should be interpreted with caution.

Limitations: First, more precise analyses stratified by other covariates, such as age, sex, environmental factors and TNM stage, could not be performed due to lack of the individual data. Second, even after pooling all existing studies, the sample size in our study was relatively small; this might have led to statistical bias in the evaluation of the association between the miR-124 rs531564 polymorphism and cancer risk, especially in the subgroup analyses. Third, all studies included in this meta-analysis were from Asia. Therefore, the limitation of the ethnic population enrolled indicates that interpretation of the associations between the SNP and the susceptibility to cancer should be made cautiously. Last, gene-gene and gene-environment interactions were not considered, which might alter the associations between miRNA gene polymorphisms and cancer. Studies with no statistically significant results often have less chance for publication.

In conclusion, our meta-analysis suggests that the miR$124 \mathrm{rs} 531564 \mathrm{G}$ allele is associated with decreased cancer risk in the Chinese population; however, this findings needs to be evaluated further in larger samples.

\section{Acknowledgements}

This work was supported by grants from the National Natural Science Foundation of China (81272323), the Natural Science Foundation of Jiangsu Province (BK2012590), the Technology Project of Changzhou Social Development (CE20125019, CE20125024, CE20135044) and the key project of the Changzhou Health Bureau (ZD201201, ZD201307, ZD201303).

\section{References}

Ahn DH, Rah H, Choi YK, et al (2013). Association of the miR146aC $>$ G, miR-149T>C, miR-196a2T $>C$, and miR-499A $>$ G polymorphisms with gastric cancer risk and survival in the Korean population. Mol Carcinog, 52, 39-51.

Ambros V (2004). The functions of animal microRNAs. Nature, 431, 350-5.

Bartel DP(2009). MicroRNAs: target recognition and regulatory functions. Cell, 136, 215-33.

Calin GA, Croce CM (2006). MicroRNA signatures in human cancers. Nat Rev Cancer, 6, 857-66.

DerSimonian R, Laird N (1986). Meta-analysis in clinical trials. Control Clin Trials, 7, 177-88.

Dikeakos $P$, Theodoropoulos G, Rizos S, et al (2014). Association of the miR-146aC $>$ G, miR-149T $>C$, and miR196a2T>C polymorphisms with gastric cancer risk and survival in the Greek population. Mol Biol Rep, 41, 1075-80.

Ebert MS, Sharp PA (2012). Roles for microRNAs in conferring robustness to biological processes. Cell, 149, 515-24.

Egger M, Davey Smith G, Schneider M, et al (1997). Bias in meta-analysis detected by a simple, graphical test. $B M J$, 315, 629-34.

Esquela-Kerscher A, Slack FJ (2006). Oncomirs - microRNAs with a role in cancer. Nat Rev Cancer, 6, 259-69.

Fabian MR, Sonenberg N (2012). The mechanics of miRNAmediated gene silencing: a look under the hood of miRISC. Nat Struct Mol Biol, 19, 586-93.

Furuta M, Kozaki KI, Tanaka S, et al (2010). miR-124 and miR-203 are epigenetically silenced tumor-suppressive microRNAs in hepatocellular carcinoma. Carcinogenesis, 31, 766-76.

Gao LB, Bai $P$, Pan XM, et al (2011). The association between two polymorphisms in pre-miRNAs and breast cancer risk: a meta-analysis. Breast Cancer Res Treat, 125, 571-4.

Hatley ME, Patrick DM, Garcia MR, et al (2010). Modulation of K-Ras-dependent lung tumorigenesis by MicroRNA-21. Cancer Cell, 18, 282-93.

He L, Hannon GJ (2004). MicroRNAs: small RNAs with a big role in gene regulation. Nat Rev Genet, 5, 522-31.

$\mathrm{Hu}$ CB, Li QL, Hu JF, et al (2014). miR-124 inhibits growth and invasion of gastric cancer by targeting ROCK1. Asian Pac $J$ Cancer Prev, 15, 6543-6.

Jansson MD, Lund AH (2012). MicroRNA and cancer. Mol Oncol, 6, 590-610.

Ji T, Zheng ZG, Wang FM, et al (2014). Differential microRNA expression by Solexa sequencing in the sera of ovarian cancer patients. Asian Pac J Cancer Prev, 15, 1739-43.

Jinushi T, Shibayama Y, Kinoshita I, et al (2014). Low expression levels of microRNA-124-5p correlated with poor prognosis in colorectal cancer via targeting of SMC4. Cancer Med, 3, 1544-52.

Kong YW, Ferland-McCollough D, Jackson TJ, et al (2012). 
microRNAs in cancer management. Lancet Oncol, 13, 249-58.

Krol J, Loedige I, Filipowicz W (2010). The widespread regulation of microRNA biogenesis, function and decay. Nat Rev Genet, 11, 597-610.

Kutanzi KR, Yurchenko OV, Beland FA, et al (2011). MicroRNA-mediated drug resistance in breast cancer. Clin Epigenetics, 2, 171-85.

Li L, Luo J, Wang B, et al (2013). Microrna-124 targets flotillin-1 to regulate proliferation and migration in breast cancer. $\mathrm{Mol}$ Cancer, 12, 163.

Li W, Zang W, Liu $P$, et al (2014). MicroRNA-124 inhibits cellular proliferation and invasion by targeting Ets-1 in breast cancer. Tumour Biol, 35, 10897-904.

Lian H, Wang L, Zhang J (2012). Increased risk of breast cancer associated with CC genotype of Has-miR-146a Rs2910164 polymorphism in Europeans. PLoS ONE, 7, 31615.

Link A, Kupcinskas J, Wex T, et al (2012). Macro-role of microRNA in gastric cancer. Dig Dis, 30, 255-67.

Mantel N, Haenszel W (1959). Statistical aspects of the analysis of data from retrospective studies of disease. J Natl Cancer Inst, 22, 719-48.

Pasquinelli AE (2012). MicroRNAs and their targets: recognition, regulation and an emerging reciprocal relationship. Nat Rev Genet, 13, 271-82.

Peng XH, Huang HR, Lu J, et al (2014). MiR-124 suppresses tumor growth and metastasis by targeting Foxq1 in nasopharyngeal carcinoma. Mol Cancer, 13, 186.

Pritchard CC, Cheng HH, Tewari M (2012). MicroRNA profiling: approaches and considerations. Nat Rev Genet, 13, 358-69.

Ruan K, Fang X, Ouyang G (2009). MicroRNAs: novel regulators in the hallmarks of human cancer. Cancer Lett, 285, 116-26.

Suarez Y, Sessa WC (2009). MicroRNAs as novel regulators of angiogenesis. Circ Res, 104, 442-54.

Tong N, Xu B, Shi D, et al (2014). Hsa-miR-196a2 polymorphism increases the risk of acute lymphoblastic leukemia in Chinese children. Mutat Res Fundam Mol Mech Mutagen, 759, 16-21.

Wan HY, Li QQ, Zhang Y, et al (2014). MiR-124 represses vasculogenic mimicry and cell motility by targeting amotL1 in cervical cancer cells. Cancer Lett, 355, 148-58.

Wang J, Bi J, Liu X, et al (2012). Has-miR-146a polymorphism (rs2910164) and cancer risk: a meta-analysis of 19 casecontrol studies. Mol Biol Rep, 39, 4571-9.

Woolf B (1955). On estimating the relation between blood group and disease. Ann Hum Genet, 19, 251-3.

Wu H, Zhang J (2014). miR-124 rs531564 polymorphism influences genetic susceptibility to cervical cancer. Int $J$ Clin Exp Med, 7, 5847-51.

Xiong X, Cheng J, Liu X, et al (2014). [Correlation analysis between miR-124 rs531564 polymorphisms and susceptibility to cervical cancer]. Nan Fang Yi Ke Da Xue Хие Bao, 34, 210-3.

Yin J, Wang X,Zheng L, et al (2013). Hsa-miR-34b/c rs4938723 $\mathrm{T}>\mathrm{C}$ and hsa-miR-423 rs6505162 $\mathrm{C}>\mathrm{A}$ polymorphisms are associated with the risk of esophageal cancer in a Chinese population. PLOS ONE, $8,80570$.

Zhang J, Huang X, Xiao J, et al (2014a). Pri-miR-124 rs531564 and pri-miR-34b/c rs4938723 polymorphisms are associated with decreased risk of esophageal squamous cell carcinoma in Chinese populations. PLOS ONE, 9, 100055.

Zhang T, Wang J, Zhai X, et al (2014b). MiR-124 retards bladder cancer growth by directly targeting CDK4. Acta Biochim Biophys Sin (Shanghai), 46, 1072-9. 